\title{
CUIDADOS PALIATIVOS EN EL DOMICILIO
}

\section{Hugo Antonio Fornells}

Médico especialista en Clínica Médica y Oncología Clínica

Miembro fundador de la Asociación Argentina de Medicina y Cuidados Paliativos Ex Presidente de la Asociación Argentina de Medicina y Cuidados Paliativos 1996/98. Director del Módulo de Medicina Paliativa de la I Cátedra de Clínica Médica de la Universidad Nacional de Rosario Coordinador del área de Cuidados Paliativos del SID (Servicio de Internación Domiciliaria)

Coordinador del área Cuidados Paliativos y tratamiento del dolor del sanatorio Británico de Rosario.

Correspondencia : Dr. Hugo Antonio Fornells. Avenida de la Libertad 370, $6^{\circ}$. (2000) Rosario -Argentina. E-mail: fornells@ satlink.com 



\title{
CUIDADOS PALIATIVOS EN EL DOMICILIO
}

\author{
Hugo Antonio Fornells
}

\section{Resumen}

La atención domiciliaria en el área de los Cuidados Paliativos requiere una coordinación entre el sistema de salud regional, las instituciones de internación hospitalaria y el equipo de trabajo domiciliario. Existen requisitos indispensables para que el paciente pueda permanecer en su casa, cumpliendo un rol principal la familia y el entorno social. No sólo se benefician el paciente y su familia sino también el sistema de salud, ya que se evitarán internaciones hospitalarias largas y de alto costo en hospitales que están más preparados para curar que para cuidar a sus pacientes. Hemos demostrado un apropiado control de síntomas de la persona enferma y logrado una alta conformidad de la familia cuando se realizan los cuidados paliativos a través de un sistema organizado. Se describen factores que contribuyen a un incorrecto cuidado domiciliario: inapropiada transición hospital-domicilio; insuficiente alivio del dolor y otros síntomas; mala comunicación e inadecuado soporte familiar. Los principios de la ética: beneficencia, no maleficencia, autonomía, justicia y equidad, algunas veces más teóricos que prácticos en la medicina moderna, se evidencian en los cuidados paliativos domiciliarios. La tarea no es simple, y se propone un cambio de política sanitaria y una actitud diferente de los profesionales de la salud, aceptando la incurabilidad de algunas enfermedades y las necesidades del enfermo terminal.

PALABRAS - CLAVE: Atención domiciliaria - Cuidados paliativos - Trabajo en equipo - Enfermo terminal.

\section{Resumo}

A atenção domiciliar referente à Cuidados Paliativos requer coordenação entre o sistema regional de saúde, o sistema de internação hospitalar e a equipe de atendimento domiciliar propriamente dita. Existem pré-requisitos familiares e sociais indispensáveis para que o paciente possa permanecer em sua residência. São beneficiários desses programas: o paciente e sua família e, especialmente, o sistema de saúde, pois reduzem-se internações com seus elevados custos hospitalares, já que essas instituições estão mais preparadas para curar enfermidades do que, propriamente, cuidar das pessoas doentes. Tem-se demonstrado que os serviços de cuidados paliativos eficientes proporcionam controle dos sintomas dos pacientes e oferecem conforto aos familiares dos mesmos. Descrevem-se, também, os fatores que contribuem para uma inadequada assistência domiciliar: transferência inapropriada do hospital para a residência; insuficiente alívio da dor e outros padecimentos do enfermo; relacionamento equipe de saúde-enfermofamília insatisfatório; inadequado suporte familiar. Os cuidados paliativos domiciliares permitem tornar práticos os, com frequência, teóricos princípios da beneficência, nãomaleficência, autonomia, justiça e equidade. A tarefa não é simples. O que se pretende é uma mudança na política sanitária, assim como, uma nova percepção pelos profissionais de saúde das reais necessidades do paciente terminal e da incurabilidade de muitas enfermidades. 


\section{Abstract}

Domiciliary assistance in the context of Palliative Care demands a coordinated action between the regional health system, hospitalizing institutions and home care team. Basic requisites must be complied with when considering the patient's staying at home, playing a major role in this setting, the family and the social environment. Not only the patient and his/her family are benefited, but also the health system, since high-cost and lengthy hospitalizations -in health institutions best prepared to cure than to take care of patients - are reduced. It has been proved that well-organized and efficient palliative care provides an adequate control of symptoms in terminally ill patients and comfort to their families. Some factors implying an unsatisfactory home health care are described: inappropriate home-hospital transition; insufficient pain and other symptoms relief; poor communication and inadequate family support. Home palliative care turns into practical the frequently theoretical ethical principles of beneficence, non-maleficence, autonomy, justice and equity. The task is by no means simple . Changes in health care policies are proposed and a new attitude and perception on the part of health care professionals is encouraged in terms of recognizing and accepting terminally ill patients' real needs and the incurability of certain diseases.

KEY-WORDS: Home health-care; Palliative Care; Teamwork; Terminally ill.

\section{Résumé}

L'attention à domicile dans le domaine des soins palliatifs precise d'une coordination entre le système de santé regionale, les institutions hospitaliers et l'équipe de travail domiciliare. Il existe des conditions pour que le patient puisse rester à la maison, en accomplissant la famille et l'entourage un rôle très important. Ce type d'attention ne bénéfice pas seulement la famille et le patient, mais aussi le système de santé puisqu'on évitera des séjours hospitaliers long et chèrs, dans des institutions plus préparés à guérir qu'à soigner leurs malades. Nous avons montré que quand les soins palliatifs se développent à travers d'un système organisé, d'un control adéquat de symtômes du malade on atteint une haute conformité de la famille. On identifie des facteurs que contribuent à des soins palliatifs inadéquats: transition hospitalière inapropiée; soulagement insuffisant de la souffrance et d'autres symtômes; mauvaise communication et soutien inadéquat de la famille. Les principes de l' éthique: bienfaisance, non malfaisance, autonomie, justice et équité, parfois plus théoriques que pratiques dans la médicine moderne, deviennent évidents dans les soins palliatifs à domicile. La tâche n'est pas facile et on propose un changement de la politique de santé et une attitude différente des professionels de la santé, en acceptant l'incurabilité de quelques maladies et les besoins du malade terminal.

MOTS CLÉS: Attention à domicile; Soins palliatifs; Travail en équipe; Malade terminal. 


\section{Introducción}

Conjugar los cuidados paliativos con la atención domiciliaria es una tarea que requiere vocación de servicio, organización del sistema de salud, y conocimientos científicos adecuados y actualizados. Los principios de la ética deberían aplicarse siempre - y más que nunca- en la atención clínica de personas que cursan el estadio terminal de una enfermedad y cuya expectativa de vida es relativamente corta. Es en esos momentos cuando el paciente y su familia transitan por situaciones que afectan profundamente los aspectos psicológicos y emocionales. Tal vez sea ésta la primera vez que se enfrentan a una situación de muerte, y si bien la muerte es algo natural y estadísticamente la cosa más normal del mundo, cuando ésta se introduce en nuestras vidas, uno no la puede aceptar como un simple hecho biológico. En general, por más que logremos admitirla, puede llegar a ser un hecho caótico que va a producir confusión y desorden en nuestra existencia. Y la desesperación y el miedo llevan en muchas oportunidades a pacientes y sus familias a buscar alternativas de tratamientos promocionados como una opción a la medicina standard. Éstas, además de no reunir ninguno de los criterios científicos, pueden ocasionar daño y en general forman parte de un negocio no ético que aprovecha, para lograr su acción, la desesperación y desilusión de personas que están sufriendo.

Los principios de la ética, algunas veces enunciados en forma teórica y en ocasiones no llevados a la práctica en la medicina moderna, forman parte de la política de salud y deben estar incluidos en todo momento en la atención de la persona terminalmente enferma, más aún en el cuidado domiciliario. Actuar siempre en beneficio del enfermo, sin incurrir en encarnizamientos terapéuticos que producirán daño al mismo, preservando su autonomía, es decir la capacidad para tomar decisiones, dentro de un régimen de justicia y equidad (derecho a ser bien aten- dido y a que no haya diferencias en la provisión de servicios) debe ser práctica diaria dentro del sistema de atención domiciliaria.

La definición de Cuidados Paliativos (1) refiere a la atención activa, global e integral de las personas que padecen una enfermedad avanzada, progresiva e incurable y de sus familias, cuyos objetivos básicos consisten en el control del dolor y demás síntomas, el apoyo emocional al enfermo y su familia y preocupación por su bienestar y calidad de vida. De tal definición se desprende que este tipo de cuidados se ofrecerá a todas las personas con diferentes enfermedades en un estadio avanzado e incurable. Lo cierto es que actualmente los servicios de cuidados paliativos se ocupan en mayor medida del cáncer avanzado y en menor proporción de otras enfermedades terminales que incluyen patologías neurológicas, respiratorias, infecciosas y cardiovasculares. Tal vez esto sea así por la alta incidencia del cáncer, la baja curabilidad del mismo, el grave deterioro funcional que va produciendo a quien lo padece y el estrés psicológico del paciente y su familia. Además, la Organización Mundial de la Salud recomienda que en los Programas Nacionales de Control del Cáncer se realicen Cuidados Paliativos, incluyendo el cuidado domiciliario (2). Es también notable observar cómo en la década del 90, año tras año, la Sociedad Americana de Oncología Clínica se ocupa cada vez más de los Cuidados Paliativos, en Oncología. Esto es evidente, ya que en la Reunión Anual de esta Sociedad, a la que asisten miles de oncólogos de todo el mundo, se presentan progresivamente más trabajos, talleres y se dictan más conferencias de Medicina y Cuidados Paliativos.

\section{Lugar de atención del paciente con enfermedad terminal}

Estudios realizados en el Reino Unido (3), en 1993, sobre una población de pacientes con enfermedad terminal y sus familiares, 
demostraron que un elevado porcentaje de los mismos prefería pasar la última etapa de su vida en su hogar. Otros estudios muestran que de los enfermos fallecidos, el $29 \%$ lo hicieron en su domicilio y el resto en el hospital. De éstos, el 50\% hubieran preferido permanecer en su domicilio y sus cuidadores hubieran deseado lo mismo si el sistema de salud fuera más competente para tal eventualidad (4).

\section{Concepto de Hospice}

Los primeros hospices para el cuidado de pacientes no curables, terminales, surgieron a fines del siglo XIX y a principio del siglo XX, en Francia y Gran Bretaña. Estas instituciones atendían caritativamente en un lugar físico a los pacientes. En 1967, la Dra. Cicely Saunders funda el St. Christopher Hospice, en Londres, donde se comienza a atender a la persona terminalmente enferma con los conceptos de la medicina científica, sin olvidarse de su componente humano, haciendo hincapié en el tratamiento del dolor.

El concepto actual de hospice que existe en el Reino Unido (5) y que se ha extendido a otros lugares del mundo, no es ya el de un edificio donde se atienden enfermos terminales. Más bien es una filosofía de trabajo, cuya base está en la aceptación y afirmación de los valores personales del paciente. Sus pilares son el alivio de síntomas y el soporte psicosocial, junto al trabajo en equipo, debiéndose también reconocer ciertos aspectos como la flexibilidad, evitando actitudes autoritarias, respetando al paciente como persona, ayudando a restaurar la creatividad del paciente y mantener su autoestima y considerando circunstancias que muchas veces olvida la medicina moderna, como lugares de internación agradables con hospitales de día con cuadros, flores, música, libros, que seguramente ayudarán a atender las necesidades espirituales del paciente.

\section{Atención domiciliaria: beneficios}

\section{Para el paciente (6)}

Se encuentra dentro de un ambiente conocido, manteniendo su intimidad, pudiendo realizar ciertas tareas laborales, continuar con sus hábitos y algunos de sus hobbies. La alimentación es más variada y los horarios no son rígidos.

\section{Para la familia}

Logra mayor satisfacción por participar activamente en el cuidado. Puede realizar los cuidados con más tranquilidad, sin apuros. Siente que respeta la voluntad del paciente de permanecer en el domicilio. Se previene el duelo patológico.

\section{Para el sistema de salud}

Se disminuyen internaciones hospitalarias largas y de alto costo. Se evita realizar tratamientos innecesarios, disminuyendo la posibilidad de caer en encarnizamientos terapéuticos. En sistemas sanitarios deficitarios, como ocurre en muchos países latinoamericanos, el ahorro económico que se produciría desplazando internaciones hospitalarias hacia el sector de atención domiciliaria continua, seguramente sería importantísimo. Este ahorro de divisas permitiría una mayor eficiencia en la salud pública y honorarios adecuados para los integrantes del equipo de cuidados paliativos en domicilio. Es difícil comprender por qué obras sociales estatales en Argentina pagan un tratamiento de quimioterapia equivalente a $\$ 3.000$, que no traerá beneficios a un paciente en estadio terminal, pero no reconocen los cuidados paliativos domiciliarios.

\section{Requisitos necesarios para que el paciente permanezca en domicilio}

Es necesario que el paciente desee estar en su casa, que tenga un entorno social y fa- 
miliar adecuado y que su familia lo pueda atender. Se requiere de un equipo sanitario de trabajo apto para realizar cuidados paliativos domiciliarios. Por último, el sistema de salud debería reconocer la prestación domiciliaria del equipo.

\section{Funcionamiento del equipo de cuidados paliativos domiciliarios del S.I.D. (Servicio de Internación Domiciliaria) Rosario, Argentina (7)}

El equipo de Cuidados Paliativos está formado por un coordinador médico, dos médicos de terreno, dos psicólogos, dos enfermeros, dos kinesiólogos y una nutricionista. Todas las semanas se realiza una reunión del grupo donde se analizan todos los pacientes que están siendo asistidos, los problemas que surgen en control de síntomas y aspectos psicosociales y las nuevas necesidades que se presentan. Estas reuniones sirven también para afianzar conocimientos del área, ya que se actualizan temas de medicina y cuidados paliativos. Los profesionales del equipo, más allá de la reunión semanal, presentan una comunicación fluida entre sí, telefónica y a través de la evolución diaria que se asienta en la Historia Clínica del paciente, que permanece en el domicilio.

El S.I.D. ofrece además la posibilidad de realizar análisis de laboratorio en domicilio, radiografías y medicina transfusional, cuando se requiere. También es posible consultar a otros profesionales, por ejemplo neurólogo, neumonólogo, que concurren al domicilio del paciente.

Los costos del servicio son, la mayoría de las veces, cubiertos por la obra social, lo cual no implica ningún gasto para el paciente. Aún no son muchas las obras sociales prepagas que aceptan el cuidado paliativo domiciliario, pero debido a gestiones periódicas que realizamos con personal jerárquico de las mismas, cada vez son más los sectores de la población hacia quienes es posible brindar atención domiciliaria.
Uno de los principios de la ética es el de la justicia. La distribución justa de los recursos médicos va a ser más probable, cuando se acepten los límites de la medicina, la incurabilidad de ciertas enfermedades y la mortalidad del ser humano. Es nuestra misión hablar todos estos temas con las empresas proveedoras de asistencia sanitaria y demostrarles que no hay nada más justo que un cuidado domiciliario adecuado para el paciente terminal y su familia, y que todos consigamos tener una muerte digna cuando ésta naturalmente llegue.

\section{Modo de trabajo del equipo de Cuidados Paliativos}

Cuando un paciente está por internarse en el domicilio, es visitado por el coordinador médico, quien evalúa cuáles son las necesidades del paciente en ese momento y programa la periodicidad de las visitas de los diferentes profesionales. Si bien las visitas son programadas, los pacientes pueden comunicarse en forma directa con cualquiera de los profesionales cuando lo crean necesario, y solicitar visitas extras. Al domicilio se lleva un maletín en cuyo interior se encuentra, bajo llave, un set básico de medicamentos de emergencia, además de los fármacos necesarios para cada paciente en particular. La historia clínica, donde se asentará cada visita profesional, la evaluación, los controles realizados y las indicaciones, se hallan igualmente dentro del maletín, al cual tendrán acceso los miembros del equipo. Si se requiere, también se provee cama ortopédica, tubo de oxígeno, nebulizador, pie de suero. Las obras sociales que realizan convenios para atención de cuidados paliativos en domicilio brindan, además, la posibilidad de estar en contacto con servicios de emergencia médica. Solicitamos al familiar que si es necesaria una atención de emergencia, una vez que lleguen profesionales médicos y paramédicos al domicilio, se comuniquen éstos con nosotros para que los 
interioricemos de la situación clínica del paciente. En nuestra experiencia son muy pocas las veces que se requiere la intervención de servicios de emergencia. Esto probablemente ocurre porque actuamos de manera proactiva, anticipándonos a los potenciales problemas, y a la educación que se realiza con el paciente y la familia.

\section{Elementos necesarios para la atención domiciliaria}

Dentro de la valija, que permanece en el domicilio y al cual tienen acceso los profesionales encargados del cuidado del paciente y el familiar cuidador principal se encuentra:

- Butterfly no 21, 23 y 25 (estos dos últimos para medicación o hidratación subcutánea).

- Jeringas de diferentes tamaños, para cargar y aplicar medicación (algunas veces se dejan preparadas dosis de medicamentos que aplicará el familiar).

- Ampollas de agua destilada y solución fisiológica para diluir medicación.

- Solución fisiológica y Dextrosa al 5\% (para comenzar una hidratación si es necesario).

- Perfuss $n^{\circ} 1$ (para conectar el sachet de hidratación al butterfly).

- Material para esterilizar piel (gasas y soluciones antisépticas).

- Medicamentos inyectables que pueden realizarse por vía subcutánea (cl. Morfina del 1 al $4 \%$ en frascos multidosis, dexametasona, diclofenac, ketorolac, 1hioscina, metoclopramida, haloperidol fenobarbital, clorpromazina).

- Historia clínica. Con todos los datos del paciente y hojas de evolución donde los profesionales asientan la evaluación y la práctica que se le realiza al paciente. Presenta una hoja para evaluación periódica de síntomas adaptada del ESAS (Edmonton Symptom Assesment System), una hoja para evaluación psicológica, una hoja para evaluación nutricional, una hoja para evaluación kinesiológica y una hoja de enfermería (además de la evaluación, asienta allí el personal de enfermería los medicamentos utilizados para el control interno y de la obra social). En próximas historias clínicas incorporaremos una sección para el minimental test (evaluación cognitiva).

\section{Utilidad de la internación domiciliaria: análisis realizado en 1998 en Rosario, Argentina (8)}

Entre 1996 y 1998, cuarenta y seis pacientes con cáncer en fase terminal fueron atendidos por el equipo de S.I.D. Esta población no representa el total de pacientes atendidos durante ese período, sino el grupo que pudo ingresar a este sistema organizado porque lo autorizó su obra social.

Al momento de realizar este estudio, cuarenta y un pacientes habían ya fallecido y cinco estaban vivos. Entre los fallecidos, veintiocho $(68,3 \%)$ lo hicieron en domicilio, mientras que trece $(31,7 \%)$ murieron internados en el sanatorio. El motivo que llevó a estos pacientes a la internación fue la mayoría de las veces el colapso familiar por agotamiento psicofísico y, en menor medida, la dificultad en el control de síntomas. El número promedio de síntomas por paciente al momento del ingreso al servicio fue 10,5 con un rango de 6 a 13 síntomas. Los más frecuentes fueron: Astenia (92\%), Anorexia (88\%), Dolor (78\%), Disnea (65\%), Depresión $(61 \%)$, Náuseas y vómitos $(58 \%)$. Tanto la frecuencia como el número de síntomas concuerda con lo descripto por otros autores en pacientes en fase terminal de enfermedad oncológica. El promedio de llamadas por paciente a un servicio de emergencias fue de 2,8, con un rango de 0 a 8 llamadas. El promedio de visitas realizadas por semana por parte del médico fue de 3,1 visitas, con un rango de 2 a 9 visitas. 
Luego de fallecido el paciente y previa comunicación con la familia se envió una encuesta a 36 personas, ya que 5 familiares no fueron hallados y respondieron el test 32 familiares $(88,8 \%)$. El test de conformidad fue diseñado por nuestro grupo. Existen algunos tests de evaluación con este fin, pero no hay ninguno que se considere estándar. Se evaluaron 5 ítems, que fueron:

1. Control de síntomas

2. Contención familiar

3. Comunicación equipo-familia-paciente

3. Alivio del sufrimiento

4. Repercusión económica.

La conformidad se evaluó a través de una escala numérica de 0 a 6 , donde 0 representaba máxima disconformidad y 6 máxima conformidad. Con respecto al ítem económico, 0 representaba máxima repercusión económica y 6 mínima. Se aclaró que la respuesta debía ser anónima.

El score promedio obtenido en cada uno de los ítems fue el siguiente:

1. Control de síntomas: 5,6

2. Contención familiar: 5,6

3. Comunicación equipo-familia-paciente: 5,7

4. Alivio de sufrimiento: 5,4

5. Repercusión económica: 5,7

Si bien consideramos que un proceso penoso, como es la pérdida de un ser querido, no puede ser medido con una escala numérica, la uniformidad de los resultados en todos los ítems evaluados nos lleva a pensar que el grado de conformidad por parte de la familia fue alto. Estos resultados nos permiten estar satisfechos, ya que el cuidado paliativo, por definición, es aquel que se realiza no sólo al paciente enfermo sino también a sus familiares, y consideramos que un cuidado paliativo adecuado disminuirá la incidencia de duelo patológico de los familiares.

\section{Factores que contribuyen a un inadecuado cuidado domiciliario}

\section{Interface hospital-domicilio inadecuada (9)}

Es muy frecuente que la auditoría del sistema de salud solicite el alta médica de un paciente terminal, ya que los costos para el sistema sanitario son elevados. Esta acción puede precipitar una externación, no existiendo una atención domiciliaria preparada. Por tal razón es necesario ir programando paulatinamente el cuidado domiciliario, mientras el paciente está aún internado. Es decir, que exista un equipo de atención que conozca al paciente y un adecuado soporte familiar que haga posible mantener comunicados a la familia, el equipo tratante y el médico de cabecera del hospital.

\section{Falta de habilidad en manejo de dolor y control de síntomas}

Muchas veces el control domiciliario recae sobre médicos jóvenes con poca formación y baja experiencia en evaluación y tratamiento de síntomas. Lo mismo ocurre con el personal de enfermería que no ha sido capacitado para el manejo del paciente terminal. Con cursos universitarios, considerando especialmente los aspectos prácticos y con el apoyo de profesionales expertos en Cuidados Paliativos, es posible ir mejorando esta situación.

\section{Comunicación inadecuada}

La falta de entrenamiento para adquirir habilidades en comunicación con el paciente y la familia es un factor preponderante que produce inadecuado control domiciliario. También es fundamental que los miembros del equipo se comuniquen entre sí periódicamente, ya que el trabajo no debe ejecutarse en forma paralela sino de manera conjunta. 


\section{Inadecuado soporte familiar}

Algunas veces, el médico generalista realiza una tarea óptima con el paciente controlando adecuadamente sus síntomas y comunicándose correctamente con él. Pero se olvida de la familia. El familiar cuidador principal necesita que le informen que todo lo que él hace por su ser querido es adecuado, pero también desea que le pregunten "cómo está usted?". Necesita que le expliquen que algunas veces es conveniente contratar a una persona para que ayude en el cuidado de su familiar y que es preciso que descanse más. Solicita que el profesional se siente y escuche sus miedos, sus dudas. Tal vez esta es la primera vez en su vida que enfrenta una situación de tal naturaleza. Necesita saber cómo tiene que hablar con los chicos.

Dedicar un tiempo más a la familia redituará en mayor beneficio para una adecuada atención domiciliaria.

\section{Emergencias domiciliarias}

Si bien no es el objetivo de este artículo extenderse en este tema, es necesario realizar algunas consideraciones.

a) En Cuidados Paliativos el profesional debe ser proactivo más que reactivo, es decir, anticiparse y prevenir situaciones de crisis.

b) En el domicilio se debe contar con los fármacos necesarios para administrar ante diferentes situaciones. Si es necesario, se dispondrá también de oxígeno, nebulizador y aspirador.

c) El familiar debe saber que puede contar con un servicio de emergencias que se pondrá en contacto con el médico tratante.

d) El familiar debe haber sido instruido en la manera de actuar si el paciente presentara una convulsión, delirio, disnea, dolor incidental severo.

e) Los familiares deben saber que las emergencias son raras, pero si ocurren, estaremos preparados para actuar.

\section{Aspectos éticos en cuidado domiciliario (10)}

\section{a) Comunicación con el paciente y la familia}

En algunas culturas orientales, como en la sociedad china, por ejemplo, se admite que el paciente no debe ser informado sobre su enfermedad terminal. En Estados Unidos, el familiar no tiene derecho a interponerse en la comunicación entre el médico y el paciente, aunque él considere que lo hace para proteger a su ser querido. En la cultura latina, si bien existen diferentes posturas, consideramos que el paciente tiene el derecho a conocer sobre su enfermedad y su pronóstico y admitimos que la comunicación es parte del tratamiento. No se impondrá un diagnóstico o un pronóstico que el paciente no desee conocer. Reflexionamos y aceptamos que el problema no radica en decir o no decir una verdad en una entrevista, sino en tener una buena comunicación. No decir la verdad no significa que debamos mentir. La comunicación es un proceso y antes de hablar deberíamos aprender a escuchar, conocer qué sabe el paciente sobre su enfermedad y qué desea conocer de la misma. Con amabilidad, hablaremos con el familiar que se interpone en una comunicación, explicaremos sobre lo negativa que es la conspiración del silencio $\mathrm{y}$ que estamos preparados para tener una comunicación adecuada con el paciente.

\section{b) Aspectos espirituales y religiosos}

Si bien también puede ser difícil para un ministro de la fe estar junto a un paciente que está muriendo, no hay duda que tienen una preparación mayor que los profesionales de la salud para comprender aspectos espirituales del paciente. Además, el entrenamiento del clero en esta área ha mejorado en los últimos años con su participación activa en congresos y cursos de Cuidados Paliativos. El médico no tiene el derecho de revelar información confidencial del pa- 
ciente al sacerdote, pero puede sugerir cuán útil podría ser contactarse con él si es que ya no lo ha hecho.

\section{c) Tratar al paciente por consideración a los familiares}

Hay pocas ocasiones en las cuales el médico encuentra que hará algún tratamiento al paciente, más para beneficio de los familiares que del propio paciente. Se realizará el tratamiento si éste no implica un daño al paciente. Algunos ejemplos pueden ilustrar esta situación. El paciente que está agonizando, inconsciente y tiene abundantes secreciones bronquiales, que están produciendo una "muerte ruidosa". Si bien el paciente no tiene conciencia de la situación, ésta es vista por los familiares como asfixia o dificultad respiratoria. No es desacertado realizar una hioscina subcutánea para disminuir las secreciones bronquiales y confortar a los parientes. En un paciente con diagnóstico reciente de cáncer de pulmón con múltiples metástasis cerebrales, que presenta convulsiones frecuentes y morirá en pocos días, no es inaceptable realizar un tratamiento con corticoides, con el objeto de mejorar los síntomas temporariamente y que viva unas semanas más, ya que los familiares no están aún preparados para la muerte inminente, y así podrán disfrutar los últimos días con él.

No es aceptable prescribir una mayor dosis de analgésicos a un paciente consciente, porque los familiares refieren que presenta más dolor de lo que realmente él relata que tiene. Diversos estudios han demostrado que los familiares perciben mayor nivel de dolor y ansiedad que la que realmente manifiesta el paciente.

\section{d) Sedación del paciente a pedido de los familiares (11)}

Si el paciente no desea mayor sedación es éticamente incorrecto realizarla a pedido del familiar. Muchas veces, algún familiar considera que han visto suficiente y ya no pueden tolerar más preguntas y no pueden aceptar más sufrimiento. Nos ocuparemos de sus necesidades, pero sedar al paciente sólo por deseo del familiar, es incorrecto.

\section{e) Mantener la vida a cualquier costo}

La Medicina Paliativa se focaliza más en calidad que en cantidad de vida. Cuando una terapia sólo prolongará una agonía sin mejorar la calidad de vida (hidratación intravenosa, nutrición parenteral, respiración asistida) del paciente terminalmente enfermo, ésta será inaceptable, aun cuando la solicite el familiar. Ya ha sido demostrado que muchas veces en domicilio es más difícil para los familiares aceptar la realidad que para el paciente mismo. Algunos médicos no familiarizados con medicina paliativa se sentirán más cómodos con tratamientos activos, aunque no se hayan planteado cuál es el objetivo final. El profesional experto en cuidados paliativos deberá explicar a los familiares y a otros colegas que ciertos tratamientos son inadecuados en este estadio de la enfermedad. No se realizará determinada terapia injustificada. Eso no significa que se abandonará al paciente. Al contrario, se le seguirá cuidando adecuadamente hasta el final.

\section{Futuro de los cuidados paliativos domiciliarios en América Latina y el Caribe}

Hasta tanto no existan políticas sanitarias adecuadas en nuestros países, la atención domiciliaria dependerá de esfuerzos individuales regionales. Actualmente, se emplean recursos financieros en una gran mayoría en terapias curativas y métodos de diagnóstico altamente sofisticados. En general, los profesionales de la salud y el sistema sanitario vigente están preparados para tratamientos agudos y es vista o vivida como un fracaso la enfermedad incurable y progresiva. En 
oncología, por ejemplo, se estima que en países en vías de desarrollo se curarán menos del $30 \%$ de los pacientes con cáncer. Sin embargo, los medios económicos se vuelcan casi en exclusividad para financiar tratamientos oncológicos activos, como quimioterapia y en muy pocos casos se reconocen los tratamientos paliativos, especialmente aquellos realizados en el domicilio del paciente.

La buena noticia es que cada vez es más firme el aumento de profesionales involucrados en Cuidados Paliativos, es mayor el número de personas que solicitan atención paliativa especializada cuando "ya no hay nada más que hacer" y se va incrementando el número de cursos de pre y post-grado de Medicina Paliativa. Muchas regiones o ciudades de nuestros países ya cuentan con líderes expertos en esta área.

Tal vez un paso que nos cuesta dar es "promocionar" los Cuidados Paliativos en la población general. Hacerlos conocidos a través de los diferentes medios de difusión masiva es una cuestión necesaria, igual que tratar de terminar con la práctica no científica y muchas veces deshumanizada de tratamiento de pacientes terminales, y procurar enrolar en Cuidados Paliativos a profesionales acreditados, solidarios y empáticos con el sufrimiento.

El único objetivo de un buen Cuidado Paliativo debe ser mantener la mejor calidad de vida posible para el paciente y su familia. Justamente el espacio donde ellos eligen estar es su domicilio. Es su lugar, es donde mejor se desempeñan, es allí donde se relacionan con los suyos, mantienen sus roles y quieren vivir. Y porque quieren vivir dignamente hasta el fin, es a su domicilio donde debemos trasladarnos para asistirlos, acompañarlos. Ya muchos profesionales hemos aceptado la invitación. Es necesario que se sumen más personas a este nuevo desafío. Es imprescindible que en los países de América Latina y el Caribe haya una redistribución más lógica de los recur- sos económicos y que actuar en Cuidados Paliativos se transforme en un trabajo íntegro y que satisfaga las necesidades de los profesionales dedicados exclusivamente a esta tarea.

\section{Conclusión}

Es posible realizar Cuidados Paliativos en Domicilio, idealmente con un equipo multiprofesional de trabajo que tenga la capacidad de ocuparse de todas las áreas de la persona enferma: somática, psicológica, social y espiritual. Es necesario también incluir a la familia del paciente en el cuidado domiciliario. Los principios de la ética: beneficencia, no maleficencia, autonomía, justicia y equidad, están presentes en forma continua cuando trabajamos con pacientes terminales, sin omitir nunca el respeto por las personas y sus creencias, junto a una comunicación honesta y adecuada. Siempre debemos recordar que aún en la era de la medicina moderna y en tiempos del postmodernismo sigue vigente el viejo adagio: Curar algunas veces, aliviar a menudo, acompañar siempre.

\section{Referencias}

1. World Health Organization. Cancer Pain Relief and Palliative Care. Geneva: WHO; 1990. (Technical Report Series 804).

2. World Health Organization. Recomendations on implementing National Cancer Control Programmes. Geneva: WHO; 1992.

3. Townsend J, Frank AO, Fermont D, Dyer S, Karran O, Walgrove A, Piper M, et al. Terminal cancer care and patients preference for place of death: a prospective study. British Medical Journal 1990; 301: 415-417.

4. Spiller J, Alexander D. Domiciliary care: a comparison of views of terminally ill patients and their family caregivers. Palliative Medicine 1993; 7: 109-15.

5. Twycross RG. Care of the terminally ill patient.[editorial] International School for Cancer Care 1993; Sept. 
6. Sergi-Swinehart P. Hospice Homecare: How to get patients home and help them to stay there. Seminars in Oncology 1985; 12 (4): 461-5.

7. Fornells H, Pérez E. Cuidados Paliativos en la Argentina. En: Gómez Sancho, (ed). Medicina Paliativa en la Cultura Latina. Buenos Aires: Atlante; 1999.

8. Poggio N, Fornells H, Tatangelo M. Análisis de la utilidad de la internación domiciliaria en pacientes con cáncer en fase terminal. $B o$ letín Científico de la Asociación Argentina de Medicina y Cuidados Paliativos 1999; 5 (10).
9. Doyle D. Domiciliary palliative care. In: Doyle D, Hanks GW, MacDonald N. Oxford Textbook of Palliative Medicine. Oxford: Oxford University Press; 1998: 957-73.

10. Gómez-Batiste X, Roca J, Trelis J, Borrell R, Novelles A. Ética clínica y cuidados paliativos. En: Gómez Batiste y otros. Cuidados Paliativos en Oncología, 1996.

11. Doyle D. Ethical issues in domiciliary care. In: Doyle D, Hanks GW, MacDonald N. Oxford Textbook of Palliative Medicine. Oxford: Oxford University Press; 1998: 970-971. 